J. Korean Math. Soc. 51 (2014), No. 4, pp. 703-719

http://dx.doi.org/10.4134/JKMS.2014.51.4.703

\title{
TRAVELING WAVE SOLUTIONS IN NONLOCAL DISPERSAL MODELS WITH NONLOCAL DELAYS
}

\author{
SHUXIa PAN
}

\begin{abstract}
This paper is concerned with the traveling wave solutions of nonlocal dispersal models with nonlocal delays. The existence of traveling wave solutions is investigated by the upper and lower solutions, and the asymptotic behavior of traveling wave solutions is studied by the idea of contracting rectangles. To illustrate these results, a delayed competition model is considered by presenting the existence and nonexistence of traveling wave solutions, which completes and improves some known results. In particular, our conclusions can deal with the traveling wave solutions of evolutionary systems which admit large time delays reflecting intraspecific competition in population dynamics and leading to the failure of comparison principle in literature.
\end{abstract}

\section{Introduction}

Nonlocal dispersal is an important spatial mode modeling many practical problems, including in material sciences [1], life sciences [13] and physics theory [8]. A typical reaction model with nonlocal dispersal takes the form as follows

$$
\begin{aligned}
\frac{\partial u(x, t)}{\partial t} & =\int_{\mathbb{R}} J(x-y)[u(y, t)-u(x, t)] d y+f(u(x, t)) \\
& =:[J * u](x, t)+f(u(x, t)),
\end{aligned}
$$

where $x \in \mathbb{R}, t>0, u \in \mathbb{R}, f: \mathbb{R} \rightarrow \mathbb{R}$ is a reaction function and $J: \mathbb{R} \rightarrow \mathbb{R}$ formulates the spatial dispersal. If the time delay is concerned in (1.1), then one corresponding delay version of (1.1) is

$$
\frac{\partial u(x, t)}{\partial t}=[J * u](x, t)+f(u(x, t), u(x, t-\tau)),
$$

in which $f: \mathbb{R}^{2} \rightarrow \mathbb{R}$ and $\tau \geq 0$ is a constant. In literature, the corresponding propagation theory of nonlocal dispersal models (1.1) and (1.2) has been widely

Received June 26, 2013

2010 Mathematics Subject Classification. 35C07, 35K57, 37C65.

Key words and phrases. upper-lower solutions, asymptotic spreading, contracting rectangle, large delay.

Supported by NSF of Gansu Province of China (1208RJYA004) and the Development Program for Outstanding Young Teachers in Lanzhou University of Technology (1010ZCX019). 
studied, and most results are indexed by the traveling wave solutions and asymptotic speed of spreading. We refer to Bates et al. [2], Carr and Chmaj [3], Chen [4], Coville and Dupaigne [5, 6], Jin and Zhao [9], Li et al. [10], Pan [14], Pan et al. [15, 16], Shen and Zhang [17], Sun et al. [20], Wu and Liu [21], Xu and Weng [23], Zhang et al. [26], Zhang et al. [27] and the references therein.

Because the traveling wave solution is a special entire solution defined for all $t \in \mathbb{R}$ and the asymptotic speed of spreading involves the long time behavior of the corresponding initial value problem, then the comparison principle is one of the most important tools in these studies (to estimate these properties of propagation modes by some special auxiliary functions/equations). Of course, in (1.1), the comparison principle holds once $f$ satisfies proper continuous condition. But in (1.2), the comparison principle does not hold for many special $f$. For example, let

$$
f(u(x, t), u(x, t-\tau))=u(x, t)(1-u(x, t)-a u(x, t-\tau)), a>0 .
$$

If $\tau>0$ is large, then the comparison principle fails such that the corresponding traveling wave solutions cannot be investigated by the mentioned results. Of course, if $\tau>0$ is very small, then we can obtain the comparison principle on a proper interval in the sense of the exponential order [19], and establish the existence of monotone traveling wave solutions of (1.2) with (1.3) by Pan [14].

In this paper, we shall further consider the traveling wave solutions of (1.2) and more systems such that we can at least answer the existence/nonexistence of traveling wave solutions of (1.2)-(1.3) with large $\tau>0$. Moreover, since the delay in (1.2) cannot model the individual movements in history [7], we shall investigate the traveling wave solutions of nonlocal dispersal systems with nonlocal delays in this paper. In order to focus on mathematical idea, we consider the traveling wave solutions of the following nonlocal dispersal systems with nonlocal delays

$$
\left\{\begin{array}{l}
\frac{\partial u_{1}(x, t)}{\partial t}=d_{1}\left[J_{1} * u_{1}\right](x, t)+f_{1}\left(u_{1}(x, t),\left(K_{1} * u_{1}\right)(x, t),\left(K_{2} * u_{2}\right)(x, t)\right), \\
\frac{\partial u_{2}(x, t)}{\partial t}=d_{2}\left[J_{2} * u_{2}\right](x, t)+f_{2}\left(u_{2}(x, t),\left(K_{3} * u_{1}\right)(x, t),\left(K_{4} * u_{2}\right)(x, t)\right),
\end{array}\right.
$$

in which $x \in \mathbb{R}, t>0,\left(u_{1}, u_{2}\right) \in \mathbb{R}^{2}, d_{1}, d_{2}$ are positive constants, $J_{1}, J_{2}$ are probability functions formulating the random dispersal of individuals and satisfy the following assumptions:

(J1) $J_{i}$ is nonnegative and Lebesgue measurable for each $i=1,2$;

(J2) for any $\lambda \in \mathbb{R}, \int_{\mathbb{R}} J_{i}(y) e^{\lambda y} d y<\infty, i=1,2$;

(J3) $\int_{\mathbb{R}} J_{i}(y) d y=1, J_{i}(y)=J_{i}(-y), y \in \mathbb{R}, i=1,2$.

In (1.4), the convolutions are defined by

$$
\left(K_{i} * u_{j}\right)(x, t)=\int_{\mathbb{R}} K_{i}(x-y) u_{j}\left(y, t-\tau_{i}\right) d y, i \in I, j=1,2,
$$

hereafter $I=:\{1,2,3,4\}, \tau_{i} \geq 0$ for each $i \in I$ and $K_{i}$ satisfies 
(K1) $K_{i}: \mathbb{R} \rightarrow \mathbb{R}$ is nonnegative for all $i \in I$

(K2) $\int_{\mathbb{R}} K_{i}(x) d x=1, K_{i}(x)=K_{i}(-x), x \in \mathbb{R}, i \in I$;

(K3) for any $\lambda>0, \int_{\mathbb{R}} K_{i}(x) e^{\lambda x} d x<\infty, i \in I$;

(K4) if $\tau_{i}=0$ for $i \in\{1,4\}$, then $\int_{\mathbb{R} \backslash\{0\}} K_{i}(x) d x=1$.

To better formulate our conditions and results, we now give the main assumptions on $f_{1}, f_{2}$ in (1.4) as follows:

(F1) $f_{1}(\cdot, u, v)$ and $f_{2}(\cdot, u, v)$ are monotone decreasing in $u, v$, without loss of generality, we take the decreasing monotonicity in Sections 2-4;

(F2) there exists $M=\left(m_{1}, m_{2}\right)$ with $m_{1}>0, m_{2}>0$ such that

$$
f_{1}\left(m_{1}, 0,0\right) \leq 0, f_{2}\left(m_{2}, 0,0\right) \leq 0, f_{1}(0,0,0)=0, f_{2}(0,0,0)=0
$$

(F3) there exists $L>0$ such that

$$
\left\{\begin{array}{l}
\left|f_{1}\left(v_{1}, v_{2}, v_{3}\right)-f_{1}\left(v_{4}, v_{5}, v_{6}\right)\right|<L\left(\left|v_{4}-v_{1}\right|+\left|v_{5}-v_{2}\right|+\left|v_{6}-v_{3}\right|\right), \\
\left|f_{2}\left(v_{7}, v_{8}, v_{9}\right)-f_{1}\left(v_{10}, v_{11}, v_{12}\right)\right|<L\left(\left|v_{10}-v_{7}\right|+\left|v_{11}-v_{8}\right|+\left|v_{12}-v_{9}\right|\right)
\end{array}\right.
$$

with $0 \leq v_{1}, v_{2}, v_{4}, v_{5}, v_{8}, v_{11} \leq m_{1}$ and $0 \leq v_{3}, v_{6}, v_{7}, v_{9}, v_{10}, v_{12} \leq m_{2}$.

These conditions state some monotone, invariant and continuous conditions on the nonlinearities. In (F1), we give a monotone condition appealing to competition behavior between intra-specific and inter-specific actions in population dynamics, such a condition is given to simplify the discussion in the remainder of this paper and better display mathematical idea. The invariance condition in (F2) depends on the monotone conditions in (F1). For example, if the monotone conditions in (F1) become the quasimonotonicity ([19]): $f_{1}\left(v_{1}, v_{2}, v_{3}\right), f_{2}\left(v_{1}, v_{2}, v_{3}\right)$ are monotone decreasing in $v_{2}, v_{3} \in \mathbb{R}^{+}$, then (F2) is: there exists $M=\left(m_{1}, m_{2}\right)$ with $m_{1}>0, m_{2}>0$ such that

$$
f_{1}\left(m_{1}, m_{1}, m_{2}\right) \leq 0, f_{2}\left(m_{2}, m_{1}, m_{2}\right) \leq 0,
$$

and the discussion in Sections 2-4 can be similarly applied to such a monotone condition, see Pan et al. [15].

To obtain the existence of traveling wave solutions, we shall introduce the definition of upper and lower solutions of the corresponding traveling wave system of (1.4), which depends on the monotone condition (F1). Furthermore, we shall consider the asymptotic behavior of traveling wave solutions by the idea of contracting rectangles of the corresponding functional differential systems. These ideas were earlier used in the study of partial functional differential systems by Lin and Ruan [12]. Moreover, to illustrate our results, we shall investigate the existence and nonexistence of traveling wave solutions if (1.4) admits the following nonlinearity (see Xia et al. [22], Yu and Yuan [24])

$$
\left\{\begin{array}{l}
f_{1}=r_{1} u_{1}(x, t)\left(1-u_{1}(x, t)-a_{1}\left(K_{1} * u_{1}\right)(x, t)-b_{1}\left(K_{2} * u_{2}\right)(x, t)\right) \\
f_{2}=r_{2} u_{2}(x, t)\left(1-u_{2}(x, t)-a_{2}\left(K_{3} * u_{1}\right)(x, t)-b_{2}\left(K_{4} * u_{2}\right)(x, t)\right)
\end{array}\right.
$$

in which all the parameters are nonnegative. 
In particular, our discussion is independent of the size of $\tau=: \max _{i \in I}\left\{\tau_{i}\right\}$. Therefore, we improve some known results on the existence and asymptotic behavior of traveling wave solutions of some models, at least for the system studied in [22]. Moreover, we also present the nonexistence of nontrivial traveling wave solutions in Section 5 and we obtain the minimal wave speed of models in Xia et al. [22], Yu and Yuan [24, Model 1.3], which completes these known conclusion.

The rest of this paper is organized as follows. In Section 2, we list necessary preliminaries. The existence of traveling wave solutions of nonlocal dispersal models is studied in Section 3. In Section 4, the asymptotic behavior of traveling wave solutions is investigated by the idea of contracting rectangles. We consider the existence and nonexistence of traveling wave solutions of a delayed competitive system with nonlocal dispersal in Section 5.

\section{Preliminaries}

In this paper, we use the standard partial ordering and order intervals in $\mathbb{R}$ or $\mathbb{R}^{2}$, and apply $\|\cdot\|$ to denote the norm in $\mathbb{R}^{2}$. Define

$X=\left\{U: U\right.$ is a bounded and uniformly continuous function from $\mathbb{R}$ to $\left.\mathbb{R}^{2}\right\}$, then $X$ is a Banach space equipped with the standard supremum norm. If $\mathbf{a}, \mathbf{b} \in \mathbb{R}^{2}$ with $\mathbf{a} \leq \mathbf{b}$, then

$$
X_{[\mathbf{a}, \mathbf{b}]}=\{U \in X: \mathbf{a} \leq U(\xi) \leq \mathbf{b}, \xi \in \mathbb{R}\} .
$$

$C^{1}\left(\mathbb{R}, \mathbb{R}^{2}\right)$ is defined by

$$
C^{1}\left(\mathbb{R}, \mathbb{R}^{2}\right)=\left\{(u, v):(u, v),\left(u^{\prime}, v^{\prime}\right) \in X\right\} .
$$

Let $\mu>0$ be a constant. Denote

$$
B_{\mu}\left(\mathbb{R}, \mathbb{R}^{2}\right)=\left\{\Phi \in X: \sup _{x \in \mathbb{R}}\|\Phi(x)\| e^{-\mu|x|}<\infty\right\}
$$

and

$$
|\Phi|_{\mu}=\sup _{x \in \mathbb{R}}\|\Phi(x)\| e^{-\mu|x|} .
$$

Then $\left(B_{\mu}\left(\mathbb{R}, \mathbb{R}^{m}\right),|\cdot|_{\mu}\right)$ is a Banach space.

Definition 2.1. A traveling wave solution of (1.4) is a special solution with the form

$$
u_{1}(x, t)=\phi_{1}(\xi), u_{2}(x, t)=\phi_{2}(\xi), \xi=x+c t,
$$

where $c>0$ is the wave speed with which the wave profile $\left(\phi_{1}, \phi_{2}\right) \in C^{1}\left(\mathbb{R}, \mathbb{R}^{2}\right)$ propagates in the spatial media $\mathbb{R}$.

By the above definition, $\left(\phi_{1}, \phi_{2}\right)$ with $c>0$ must satisfy

$$
\begin{cases}c \phi_{1}^{\prime}(\xi)=d_{1}\left[J_{1} * \phi_{1}\right](\xi)+f_{1}\left(\phi_{1}(\xi),\left(K_{1} * \phi_{1}\right)(\xi),\left(K_{2} * \phi_{2}\right)(\xi)\right), & \xi \in \mathbb{R} \\ c \phi_{2}^{\prime}(\xi)=d_{2}\left[J_{2} * \phi_{2}\right](\xi)+f_{2}\left(\phi_{2}(\xi),\left(K_{3} * \phi_{1}\right)(\xi),\left(K_{4} * \phi_{2}\right)(\xi)\right), \quad \xi \in \mathbb{R}\end{cases}
$$


with

$$
\left\{\begin{array}{l}
{\left[J_{i} * \phi_{i}\right](\xi)=\int_{\mathbb{R}} J_{i}(y)\left[\phi_{i}(\xi-y)-\phi_{i}(\xi)\right] d y, i=1,2} \\
\left(K_{i} * \phi_{j}\right)(\xi)=\int_{\mathbb{R}} K_{i}(\xi-y) \phi_{j}\left(y-c \tau_{i}\right) d y, i \in I, j=1,2 .
\end{array}\right.
$$

Moreover, to model the transition process between different states, we often add proper asymptotic boundary conditions of $\left(\phi_{1}, \phi_{2}\right)$. In this paper, we require that

$$
\lim _{\xi \rightarrow-\infty}\left(\phi_{1}(\xi), \phi_{2}(\xi)\right)=(0,0), \liminf _{\xi \rightarrow \infty}\left(\phi_{1}(\xi), \phi_{2}(\xi)\right) \gg(0,0) .
$$

Since $\xi=x+c t$, then a traveling wave solution satisfying (2.2)-(2.3) formulates the simultaneously successful invasion of two competitive species in population dynamics [18]. In particular, we also assume that

(F4) there exists $E=\left(e_{1}, e_{2}\right) \gg(0,0)$ such that $f_{1}\left(e_{1}, e_{1}, e_{2}\right)=f_{2}\left(e_{2}, e_{1}, e_{2}\right)$ $=0$,

and study the following stronger condition

$$
\lim _{\xi \rightarrow-\infty}\left(\phi_{1}(\xi), \phi_{2}(\xi)\right)=(0,0), \lim _{\xi \rightarrow \infty}\left(\phi_{1}(\xi), \phi_{2}(\xi)\right)=\left(e_{1}, e_{2}\right) .
$$

Let $\beta>0$ be a constant such that

$$
\left(\beta-d_{1}\right) \phi_{1}+f_{1}\left(\phi_{1}, m_{1}, m_{2}\right),\left(\beta-d_{2}\right) \phi_{2}+f_{2}\left(\phi_{2}, m_{1}, m_{2}\right)
$$

are monotone increasing in $\phi_{1} \in\left[0, m_{1}\right], \phi_{2} \in\left[0, m_{2}\right]$, respectively.

For $\Phi=\left(\phi_{1}, \phi_{2}\right) \in X_{[\mathbf{0}, \mathbf{M}]}$, define

$$
\left\{\begin{array}{l}
H_{1}\left(\phi_{1}, \phi_{2}\right)(\xi)=\beta \phi_{1}(\xi)+d_{1}\left[J_{1} * \phi_{1}\right](\xi)+f_{1}\left(\phi_{1}(\xi),\left(K_{1} * \phi_{1}\right)(\xi),\left(K_{2} * \phi_{2}\right)(\xi)\right), \\
H_{2}\left(\phi_{1}, \phi_{2}\right)(\xi)=\beta \phi_{2}(\xi)+d_{2}\left[J_{2} * \phi_{2}\right](\xi)+f_{2}\left(\phi_{2}(\xi),\left(K_{3} * \phi_{1}\right)(\xi),\left(K_{4} * \phi_{2}\right)(\xi)\right) .
\end{array}\right.
$$

With these notations, let $P=\left(P_{1}, P_{2}\right): X_{[\mathbf{0}, \mathbf{M}]} \rightarrow X$ be

$$
\left\{\begin{array}{l}
P_{1}\left(\phi_{1}, \phi_{2}\right)(\xi)=\frac{1}{c} \int_{-\infty}^{\xi} e^{-\frac{\beta(\xi-s)}{c}} H_{1}\left(\phi_{1}, \phi_{2}\right)(s) d s \\
P_{2}\left(\phi_{1}, \phi_{2}\right)(\xi)=\frac{1}{c} \int_{-\infty}^{\xi} e^{-\frac{\beta(\xi-s)}{c}} H_{2}\left(\phi_{1}, \phi_{2}\right)(s) d s .
\end{array}\right.
$$

Then a fixed point of $P$ is a solution to (2.1), and it suffices to investigate the existence and asymptotic behavior of fixed points of $P$.

Finally, we recall some results in Jin and Zhao [9], Shen and Zhang [17]. Consider

$$
\left\{\begin{array}{l}
\frac{\partial u(x, t)}{\partial t}=d[J * u](x, t)+r u(x, t)[1-u(x, t)] \\
u(x, 0)=\chi(x), x \in \mathbb{R}
\end{array}\right.
$$

where $J$ satisfies (J1)-(J3), $d>0$ and $r>0$ are constants, and the initial value $\chi(x)$ is uniformly continuous and bounded. By [9, Theorem 2.3], we have the following comparison principle of (2.5). 
Lemma 2.2. Assume that $0 \leq \chi(x) \leq 1$. Then (2.5) admits a solution for all $x \in \mathbb{R}, t>0$. If $w(x, 0)$ is uniformly continuous and bounded, and $w(x, t)$ satisfies

$$
\left\{\begin{array}{l}
\frac{\partial w(x, t)}{\partial t} \geq(\leq) d[J * w](x, t)+r w(x, t)[1-w(x, t)], x \in \mathbb{R}, t>0 \\
w(x, 0) \geq(\leq) \chi(x), x \in \mathbb{R}
\end{array}\right.
$$

then

$$
w(x, t) \geq(\leq) u(x, t), x \in \mathbb{R}, t>0 .
$$

For $c>0, \lambda>0$, define

$$
\Theta(\lambda, c)=d\left[\int_{\mathbb{R}} J(y) e^{\lambda y} d y-1\right]-c \lambda+r .
$$

Lemma 2.3. There exists $c_{*}>0$ such that

(R1) if $c<c_{*}$, then $\Theta(\lambda, c)>0$ for any $\lambda>0$;

(R2) if $c>c_{*}$, there exist positive constants $\mu_{1}(c), \mu_{2}(c)$ such that

$$
\Theta(\lambda, c)\left\{\begin{array}{l}
=0, \lambda=\mu_{1}(c), \mu_{2}(c) \\
<0, \lambda \in\left(\mu_{1}(c), \mu_{2}(c)\right), \\
>0, \lambda \in\left(0, \mu_{1}(c) \bigcup\left(\mu_{2}(c),+\infty\right) .\right.
\end{array}\right.
$$

Lemma 2.4. Assume that $\chi(x)>0$. Then for any $c<c_{*}$, we have

$$
\liminf _{t \rightarrow \infty} \inf _{|x|<c t} u(x, t)=\limsup _{t \rightarrow \infty} \sup _{|x|<c t} u(x, t)=1 .
$$

\section{Existence of traveling wave solutions}

To investigate the existence of traveling wave solutions, we first introduce the following upper and lower solutions of (2.1).

Definition 3.1. Assume that (F1)-(F3) hold and

$$
\bar{\Phi}(\xi)=\left(\bar{\phi}_{1}(\xi), \bar{\phi}_{2}(\xi)\right), \underline{\Phi}(\xi)=\left(\underline{\phi}_{1}(\xi), \underline{\phi}_{2}(\xi)\right) \in X_{[\mathbf{0}, \mathbf{M}]}
$$

are differentiable for $\xi \in \mathbb{R} \backslash \mathbb{T}$, where $\mathbb{T}$ contains finite points of $\mathbb{R}$. If $\bar{\Phi}(\xi) \geq$ $\underline{\Phi}(\xi)$ satisfy

$\left\{c \bar{\phi}_{1}^{\prime}(\xi) \geq d_{1}\left[J_{1} * \bar{\phi}_{1}\right](\xi)+\bar{F}_{1}(\xi), c \bar{\phi}_{2}^{\prime}(\xi) \geq d_{2}\left[J_{2} * \bar{\phi}_{2}\right](\xi)+\bar{F}_{2}(\xi), \xi \in \mathbb{R} \backslash \mathbb{T}\right.$,

$\left\{c \underline{\phi}_{1}^{\prime}(\xi) \leq d_{1}\left[J_{1} * \underline{\phi}_{1}\right](\xi)+\underline{F}_{1}(\xi), c \underline{\phi}_{2}^{\prime}(\xi) \leq d_{2}\left[J_{2} * \underline{\phi}_{2}\right](\xi)+\underline{F}_{2}(\xi), \xi \in \mathbb{R} \backslash \mathbb{T}\right.$,

in which $\bar{F}_{1}(\xi), \bar{F}_{2}(\xi), \underline{F}_{1}(\xi), \underline{F}_{2}(\xi)$ are defined by

$$
\left\{\begin{array}{l}
\bar{F}_{1}(\xi)=f_{1}\left(\bar{\phi}_{1}(\xi),\left(K_{1} * \underline{\phi}_{1}\right)(\xi),\left(K_{2} * \underline{\phi}_{2}\right)(\xi)\right), \\
\bar{F}_{2}(\xi)=f_{2}\left(\bar{\phi}_{2}(\xi),\left(K_{3} * \bar{\phi}_{1}\right)(\xi),\left(K_{4} * \bar{\phi}_{2}\right)(\xi)\right), \\
\underline{F}_{1}(\xi)=f_{1}\left(\underline{\phi}_{1}(\xi),\left(K_{1} * \bar{\phi}_{1}\right)(\xi),\left(K_{2} * \bar{\phi}_{2}\right)(\xi)\right), \\
\underline{F}_{2}(\xi)=f_{2}\left(\underline{\phi}_{2}(\xi),\left(K_{3} * \bar{\phi}_{1}\right)(\xi),\left(K_{4} * \bar{\phi}_{2}\right)(\xi)\right),
\end{array}\right.
$$

then $\bar{\Phi}(\xi)$ is an upper solution while $\underline{\Phi}(\xi)$ is a lower solution of (2.1). 
We now present the main conclusion of this section.

Theorem 3.2. Assume that (J1)-(J3), (K1)-(K4) and (F1)-(F3) hold. For any fixed $c>0$, if (2.1) admits an upper solution $\bar{\Phi} \in X_{[\mathbf{0}, \mathbf{M}]}$ and a lower solution $\underline{\Phi} \in X_{[\mathbf{0}, \mathbf{M}]}$, then (2.1) has a positive solution $\Phi \in X_{[\mathbf{0}, \mathbf{M}]}$ satisfying $\underline{\Phi} \leq \Phi \leq \bar{\Phi}$.

In the remainder of this section, we shall prove the theorem by several lemmas, through which the conditions of Theorem 3.2 will be imposed. Define

$$
\Gamma=\{\Phi \in X: \underline{\Phi} \leq \Phi \leq \bar{\Phi}\} .
$$

Lemma 3.3. Assume that $\Phi=\left(\phi_{1}, \phi_{2}\right) \in \Gamma$. Then $P(\Phi) \in \Gamma$.

Proof. By (F3), $P(\Phi) \in X$. Using (F1), we have

$$
\left\{\begin{array}{l}
H_{1}\left(\phi_{1}, \phi_{2}\right)(\xi) \geq \beta \underline{\phi}_{1}(\xi)+d_{1}\left[J_{1} * \underline{\phi}_{1}\right](\xi)+\underline{F}_{1}(\xi)=: \underline{H}_{1}(\xi), \xi \in \mathbb{R}, \\
H_{1}\left(\phi_{1}, \phi_{2}\right)(\xi) \leq \beta \bar{\phi}_{1}(\xi)+d_{1}\left[J_{1} * \bar{\phi}_{1}\right](\xi)+\bar{F}_{1}(\xi)=: \bar{H}_{1}(\xi), \xi \in \mathbb{R} .
\end{array}\right.
$$

Let

$$
\underline{P}_{1}(\xi)=\frac{1}{c} \int_{-\infty}^{\xi} e^{-\frac{\beta(\xi-s)}{c}} \underline{H}_{1}(s) d s, \bar{P}_{1}(\xi)=\frac{1}{c} \int_{-\infty}^{\xi} e^{-\frac{\beta(\xi-s)}{c}} \bar{H}_{1}(s) d s, \xi \in \mathbb{R} .
$$

Then it suffices to prove that

$$
\underline{\phi}_{1}(\xi) \leq \underline{P}_{1}(\xi) \leq \bar{P}_{1}(\xi) \leq \bar{\phi}_{1}(\xi), \xi \in \mathbb{R} .
$$

In fact, let

$$
\mathbb{T}=\left\{T_{1}, T_{2}, \ldots, T_{n}\right\}
$$

and denote $T_{0}=-\infty, T_{n+1}=\infty$. If $\xi \in\left(T_{k-1}, T_{k}\right)$ with some $k \in\{1,2, \ldots, n+$ $1\}$, then

$$
\begin{aligned}
\bar{P}_{1}(\xi) & =\frac{1}{c} \int_{-\infty}^{\xi} e^{-\frac{\beta(\xi-s)}{c}} \bar{H}_{1}(s) d s \\
& =\left(\sum_{i=0}^{k-1} \frac{1}{c} \int_{T_{i-1}}^{T_{i}}+\frac{1}{c} \int_{T_{k-1}}^{\xi}\right) e^{-\frac{\beta(\xi-s)}{c} \bar{H}_{1}(s) d s} \\
& \leq\left(\sum_{i=0}^{k-1} \frac{1}{c} \int_{T_{i-1}}^{T_{i}}+\frac{1}{c} \int_{T_{k-1}}^{\xi}\right) e^{-\frac{\beta(\xi-s)}{c}}\left(c \bar{\phi}_{1}^{\prime}(s)-\beta \bar{\phi}_{1}(s)\right) \\
& =\bar{\phi}_{1}(\xi) .
\end{aligned}
$$

Using the continuity of both $\bar{P}_{1}(\xi)$ and $\bar{\phi}_{1}(\xi)$, we have

$$
\bar{P}_{1}(\xi) \leq \bar{\phi}_{1}(\xi), \xi \in \mathbb{R} \text {. }
$$

In a similar way, we can verify that

$$
\underline{\phi}_{1}(\xi) \leq \underline{P}_{1}(\xi), \xi \in \mathbb{R}
$$

and

$$
\underline{\phi}_{2}(\xi) \leq P_{2}(\Phi)(\xi) \leq \bar{\phi}_{2}(\xi), \xi \in \mathbb{R}
$$


The proof is complete.

Lemma 3.4. If $\mu<\frac{\beta}{2 c}$, then $P: \Gamma \rightarrow \Gamma$ is complete continuous in the sense of $|\cdot|_{\mu}$.

Proof. Let $\Phi=\left(\phi_{1}, \phi_{2}\right), \Psi=\left(\psi_{1}, \psi_{2}\right) \in \Gamma$. Then

$$
\begin{aligned}
& \left|H_{1}\left(\phi_{1}, \phi_{2}\right)(\xi)-H_{1}\left(\psi_{1}, \psi_{2}\right)(\xi)\right| \\
= & \mid\left(\beta-d_{1}\right) \phi_{1}(\xi)+f_{1}\left(\phi_{1}(\xi),\left(K_{1} * \phi_{1}\right)(\xi),\left(K_{2} * \phi_{2}\right)(\xi)\right) \\
& -\left(\beta-d_{1}\right) \psi_{1}(\xi)-f_{1}\left(\psi_{1}(\xi),\left(K_{1} * \psi_{1}\right)(\xi),\left(K_{2} * \psi_{2}\right)(\xi)\right) \\
& +d_{1} \int_{\mathbb{R}} J_{1}(\xi-y) \phi_{1}(y) d y-d_{1} \int_{\mathbb{R}} J_{1}(\xi-y) \psi_{1}(y) d y \mid \\
\leq & \left(\beta-d_{1}\right)\left|\phi_{1}(\xi)-\psi_{1}(\xi)\right|+d_{1} \int_{\mathbb{R}} J_{1}(\xi-y)\left|\phi_{1}(y)-\psi_{1}(y)\right| d y \\
& +\left|f_{1}\left(\phi_{1}(\xi),\left(K_{1} * \phi_{1}\right)(\xi),\left(K_{2} * \phi_{2}\right)(\xi)\right)-f_{1}\left(\psi_{1}(\xi),\left(K_{1} * \psi_{1}\right)(\xi),\left(K_{2} * \psi_{2}\right)(\xi)\right)\right| \\
\leq & \left(L+\beta-d_{1}\right)\left|\phi_{1}(\xi)-\psi_{1}(\xi)\right|+d_{1} \int_{\mathbb{R}} J_{1}(\xi-y)\left|\phi_{1}(y)-\psi_{1}(y)\right| d y \\
& +L \int_{\mathbb{R}} K_{1}(\xi-y)\left|\phi_{1}\left(y-c \tau_{1}\right)-\psi_{1}\left(y-c \tau_{1}\right)\right| d y \\
& +L \int_{\mathbb{R}} K_{2}(\xi-y)\left|\phi_{2}\left(y-c \tau_{2}\right)-\psi_{2}\left(y-c \tau_{2}\right)\right| d y,
\end{aligned}
$$

and

$$
\begin{aligned}
& \left|P_{1}\left(\phi_{1}, \phi_{2}\right)(\xi)-P_{1}\left(\psi_{1}, \psi_{2}\right)(\xi)\right| e^{-\mu|\xi|} \\
\leq & \frac{\left(L+\beta-d_{1}\right) e^{-\mu|\xi|}}{c} \int_{-\infty}^{\xi} e^{-\frac{\beta(\xi-s)}{c}}\left|\phi_{1}(s)-\psi_{1}(s)\right| d s \\
& +\frac{d_{1} e^{-\mu|\xi|}}{c} \int_{-\infty}^{\xi} e^{-\frac{\beta(\xi-s)}{c}}\left(\int_{\mathbb{R}} J_{1}(s-y)\left|\phi_{1}(y)-\psi_{1}(y)\right| d y\right) d s \\
& +\frac{L e^{-\mu|\xi|}}{c} \int_{-\infty}^{\xi} e^{-\frac{\beta(\xi-s)}{c}}\left(\int_{\mathbb{R}} K_{1}(s-y)\left|\phi_{1}\left(y-c \tau_{1}\right)-\psi_{1}\left(y-c \tau_{1}\right)\right| d y\right) d s \\
& +\frac{L e^{-\mu|\xi|}}{c} \int_{-\infty}^{\xi} e^{-\frac{\beta(\xi-s)}{c}}\left(\int_{\mathbb{R}} K_{2}(s-y)\left|\phi_{2}\left(y-c \tau_{2}\right)-\psi_{2}\left(y-c \tau_{2}\right)\right| d y\right) d s \\
=: & I_{1}+I_{2}+I_{3}+I_{4},
\end{aligned}
$$

in which the definitions of $I_{1}, I_{2}, I_{3}, I_{4}$ are clear. Furthermore,

$$
\begin{aligned}
I_{1} & =\frac{\left(L+\beta-d_{1}\right) e^{-\mu|\xi|}}{c} \int_{-\infty}^{\xi} e^{-\frac{\beta(\xi-s)}{c}}\left|\phi_{1}(s)-\psi_{1}(s)\right| d s \\
& =\frac{\left(L+\beta-d_{1}\right) e^{-\mu|\xi|}}{c} \int_{-\infty}^{\xi} e^{-\frac{\beta(\xi-s)}{c}} e^{\mu|s|}\left|\phi_{1}(s)-\psi_{1}(s)\right| e^{-\mu|s|} d s \\
& \leq \frac{|\Phi-\Psi|_{\mu}\left(L+\beta-d_{1}\right)}{c} \int_{-\infty}^{\xi} e^{-\frac{\beta(\xi-s)}{c}} e^{-\mu|\xi|} e^{\mu|s|} d s .
\end{aligned}
$$


If $\xi \leq 0$, then $-\mu|\xi|+\mu|s|=\mu(\xi-s)$. If $\xi>0, s \leq 0$, then

$$
-\mu|\xi|+\mu|s|=\mu(-\xi-s) \leq \mu(\xi-s) .
$$

If $\xi>0, s>0$, then

$$
-\mu|\xi|+\mu|s|=\mu(-\xi+s)=-\mu(\xi-s) \leq \mu(\xi-s) .
$$

Therefore, we obtain

$$
\int_{-\infty}^{\xi} e^{-\frac{\beta(\xi-s)}{c}} e^{-\mu|\xi|} e^{\mu|s|} d s \leq \int_{-\infty}^{\xi} e^{\left(\frac{c \mu-\beta}{c}\right)(\xi-s)} d s=\frac{c}{\beta-c \mu},
$$

which further implies that

$$
I_{1} \leq \frac{\left(L+\beta-d_{1}\right)}{\beta-c \mu}|\Phi-\Psi|_{\mu} .
$$

In a similar way, we have

$$
\begin{aligned}
& I_{2} \leq \frac{d_{1} \int_{\mathbb{R}} J_{1}(y) e^{\mu|y|} d y}{\beta-c \mu}|\Phi-\Psi|_{\mu}, \\
& I_{3} \leq \frac{L e^{\mu c \tau_{1}} \int_{\mathbb{R}} K_{1}(y) e^{\mu|y|} d y}{\beta-c \mu}|\Phi-\Psi|_{\mu}, \\
& I_{4} \leq \frac{L e^{\mu c \tau_{2}} \int_{\mathbb{R}} K_{2}(y) e^{\mu|y|} d y}{\beta-c \mu}|\Phi-\Psi|_{\mu} .
\end{aligned}
$$

Let

$$
L^{\prime}=\frac{L+\beta-d_{1}+d_{1} \int_{\mathbb{R}} J_{1}(y) e^{\mu|y|} d y+L e^{\mu c \tau_{1}} \int_{\mathbb{R}} K_{1}(y) e^{\mu|y|} d y+L e^{\mu c \tau_{2}} \int_{\mathbb{R}} K_{2}(y) e^{\mu|y|} d y}{\beta-c \mu},
$$

then $\left|P_{1}\left(\phi_{1}, \phi_{2}\right)(\xi)-P_{1}\left(\psi_{1}, \psi_{2}\right)(\xi)\right| e^{-\mu|\xi|} \leq L^{\prime}|\Phi-\Psi|_{\mu}$ and

$$
\sup _{\xi \in \mathbb{R}}\left|P_{1}\left(\phi_{1}, \phi_{2}\right)(\xi)-P_{1}\left(\psi_{1}, \psi_{2}\right)(\xi)\right| e^{-\mu|\xi|} \leq L^{\prime}|\Phi-\Psi|_{\mu} .
$$

After a similar discussion on $P_{2}$, we obtain the continuity of $P$ with respect to $|\cdot|_{\mu}$.

Moreover, from (F3), we can verify the equicontinuity of the mapping. By the Ascoli-Arzela lemma, for any $\varepsilon>0$ and $B>0$, we can choose a subset $\Gamma^{\prime}$ of $P(\Gamma)$ and $\Gamma^{\prime}$ contains finite functions, such that the restriction of $\Gamma^{\prime}$ on $[-B, B]$ is a finite $\varepsilon$-net of the restriction of $P(\Gamma)$ on $[-B, B]$ in the sense of the supremum norm. In particular, let $B>0$ be large such that

$$
e^{-\mu B}\left(m_{1}+m_{2}\right)<\varepsilon,
$$

then $\Gamma^{\prime}$ is a finite $\varepsilon$-net of $P(\Gamma)$ in the sense of $|\cdot|_{\mu}$. The proof is complete.

Note that $\Gamma$ is nonempty and convex, and is bounded and closed with respect to the norm $|\cdot|_{\mu}$. By Schauder's fixed point theorem and Lemmas 3.3 and 3.4, we complete the proof of Theorem 3.2. 


\section{Asymptotic behavior of traveling wave solutions}

We now investigate the asymptotic behavior of traveling wave solutions. Since the traveling wave solutions may be nonmonotonic, we cannot obtain the asymptotic behavior by the monotonicity when $\xi \rightarrow \infty$. To answer the question, we first consider the corresponding functional differential equation of (1.4) as follows

$$
\left\{\begin{array}{l}
u_{1}^{\prime}(t)=f_{1}\left(u_{1}(t), u_{1}\left(t-\tau_{1}\right), u_{2}\left(t-\tau_{2}\right)\right), \\
u_{2}^{\prime}(t)=f_{2}\left(u_{2}(t), u_{1}\left(t-\tau_{3}\right), u_{2}\left(t-\tau_{4}\right)\right), \\
u_{1}(s)=\psi_{1}(s), u_{2}(s)=\psi_{2}(s), s \in[-\tau, 0],
\end{array}\right.
$$

in which $f_{1}, f_{2}$ satisfy (F3)-(F4), and $\psi_{1}(s), \psi_{1}(s)$ are continuous for $s \in[-\tau, 0]$.

Definition 4.1. $\left[\left(r_{1}(z), s_{1}(z)\right),\left(r_{2}(z), s_{2}(z)\right)\right]$ is a contracting rectangle of (4.1) if

(C1) $r_{1}(z), s_{1}(z)$ are continuous and strictly increasing while $r_{2}(z), s_{2}(z)$ are continuous and strictly decreasing for $z \in[0,1]$;

(C2) for $z \in[0,1]$,

$$
\left\{\begin{array}{l}
r_{1}(0) \leq r_{1}(z) \leq r_{1}(1)=e_{1}=r_{2}(1) \leq r_{2}(z) \leq r_{2}(0) \\
s_{1}(0) \leq s_{1}(z) \leq s_{1}(1)=e_{2}=s_{2}(1) \leq s_{2}(z) \leq s_{2}(0)
\end{array}\right.
$$

(C3) if $a_{1}=r_{1}\left(z_{0}\right)\left(a_{2}=s_{1}\left(z_{0}\right)\right)$ for any $z_{0} \in(0,1)$ and

$$
r_{1}\left(z_{0}\right) \leq b_{1} \leq r_{2}\left(z_{0}\right), s_{1}\left(z_{0}\right) \leq b_{2} \leq s_{2}\left(z_{0}\right),
$$

then $f_{1}\left(a_{1}, b_{1}, b_{2}\right)>0\left(f_{2}\left(a_{2}, b_{1}, b_{2}\right)>0\right)$; if $a_{1}=r_{2}\left(z_{0}\right)\left(a_{2}=s_{2}\left(z_{0}\right)\right)$ for some $z_{0} \in(0,1)$ and

$$
r_{1}\left(z_{0}\right) \leq b_{1} \leq r_{2}\left(z_{0}\right), s_{1}\left(z_{0}\right) \leq b_{2} \leq s_{2}\left(z_{0}\right),
$$

then $f_{1}\left(a_{1}, b_{1}, b_{2}\right)<0\left(f_{2}\left(a_{2}, b_{1}, b_{2}\right)<0\right)$.

In Smith [19], we can find some convergence results of the initial value problem (4.1) via the technique of contracting rectangle. In what follows, we present the asymptotic behavior of traveling wave solutions of (1.4) by the contracting rectangle.

Theorem 4.2. Assume that (J1)-(J3), (K1)-(K4) and (F1)-(F4) hold. Let $\Phi=\left(\phi_{1}, \phi_{2}\right) \in X_{[\mathbf{0}, \mathbf{M}]}$ be a solution of $(2.1)$ or be a fixed point of $P$. If

$$
\left\{\begin{array}{l}
r_{1}\left(z_{0}\right) \leq \liminf _{\xi \rightarrow \infty} \phi_{1}(\xi) \leq \limsup _{\xi \rightarrow \infty} \phi_{1}(\xi) \leq r_{2}\left(z_{0}\right), \\
s_{1}\left(z_{0}\right) \leq \liminf _{\xi \rightarrow \infty} \phi_{2}(\xi) \leq \lim \sup _{\xi \rightarrow \infty} \phi_{2}(\xi) \leq s_{2}\left(z_{0}\right)
\end{array}\right.
$$

for some $z_{0} \in(0,1)$, where $r_{1}(z), s_{1}(z), r_{2}(z), s_{2}(z)$ satisfy Definition 4.1. Then

$$
\lim _{\xi \rightarrow \infty}\left(\phi_{1}(\xi), \phi_{2}(\xi)\right)=\left(e_{1}, e_{2}\right) \text {. }
$$


Proof. Were the statement false, then there exists $z_{1} \in\left[z_{0}, 1\right)$ such that

$$
\begin{array}{ll}
r_{1}\left(z_{1}\right) \leq \liminf _{\xi \rightarrow \infty} \phi_{1}(\xi), & \quad \limsup _{\xi \rightarrow \infty} \phi_{1}(\xi) \leq r_{2}\left(z_{1}\right), \\
s_{1}\left(z_{1}\right) \leq \liminf _{\xi \rightarrow \infty} \phi_{2}(\xi), & \limsup _{\xi \rightarrow \infty} \phi_{2}(\xi) \leq s_{2}\left(z_{1}\right)
\end{array}
$$

and at least one of the following equalities is true:

$$
\begin{aligned}
& r_{1}\left(z_{1}\right)=\liminf _{\xi \rightarrow \infty} \phi_{1}(\xi), \quad \limsup _{\xi \rightarrow \infty} \phi_{1}(\xi)=r_{2}\left(z_{1}\right), \\
& s_{1}\left(z_{1}\right)=\liminf _{\xi \rightarrow \infty} \phi_{2}(\xi), \quad \limsup _{\xi \rightarrow \infty} \phi_{2}(\xi)=s_{2}\left(z_{1}\right) .
\end{aligned}
$$

Without loss of generality, we assume that

$$
r_{1}\left(z_{1}\right)=\liminf _{\xi \rightarrow \infty} \phi_{1}(\xi) .
$$

Letting $\xi \rightarrow \infty$ and using the dominated convergence theorem in $P_{1}$, we obtain

$$
\begin{aligned}
& \liminf _{\xi \rightarrow \infty} \phi_{1}(\xi) \\
\geq & \frac{\beta \liminf \inf _{\xi \rightarrow \infty} \phi_{1}(\xi)+f_{1}\left(\liminf _{\xi \rightarrow \infty} \phi_{1}(\xi), \lim \sup _{\xi \rightarrow \infty} \phi_{1}(\xi), \lim _{\sup _{\xi \rightarrow \infty}} \phi_{1}(\xi)\right)}{\beta}
\end{aligned}
$$

by the monotone condition (F1), the continuous condition (F3) and the definition of $\beta$. This further indicates that

$$
f_{1}\left(\liminf _{\xi \rightarrow \infty} \phi_{1}(\xi), \limsup _{\xi \rightarrow \infty} \phi_{1}(\xi), \limsup _{\xi \rightarrow \infty} \phi_{1}(\xi)\right) \leq 0,
$$

and a contradiction occurs from (C3). The proof is complete.

In what follows, we shall give several remarks on the discussion in Sections $3-4$.

Remark 4.3. The definition of contracting rectangle is independent of the monotone condition in (F1), so we can apply the idea in Theorem 4.2 to other monotone nonlinearities.

Remark 4.4. In Section 1, we have mentioned the form of invariance condition (F2) depends on the monotone condition (F1). Moreover, the definition of upper and lower solutions also depends on (F1). In particular, if the delayed system (1.4) is quasimonotone, then we should replace (3.2) by

$$
\left\{\begin{array}{l}
\bar{F}_{1}(\xi)=f_{1}\left(\bar{\phi}_{1}(\xi),\left(K_{1} * \bar{\phi}_{1}\right)(\xi),\left(K_{2} * \bar{\phi}_{2}\right)(\xi)\right), \\
\bar{F}_{2}(\xi)=f_{2}\left(\bar{\phi}_{2}(\xi),\left(K_{3} * \bar{\phi}_{1}\right)(\xi),\left(K_{4} * \bar{\phi}_{2}\right)(\xi)\right), \\
\underline{F}_{1}(\xi)=f_{1}\left(\underline{\phi}_{1}(\xi),\left(K_{1} * \underline{\phi}_{1}\right)(\xi),\left(K_{2} * \underline{\phi}_{2}\right)(\xi)\right), \\
\underline{F}_{2}(\xi)=f_{2}\left(\underline{\phi}_{2}(\xi),\left(K_{3} * \underline{\phi}_{1}\right)(\xi),\left(K_{4} * \underline{\phi}_{2}\right)(\xi)\right) .
\end{array}\right.
$$

For other monotone condition, e.g., in the predator-prey system, we should make necessary changes in the definition of upper and lower solutions (see Yu and Yuan [25] and Zhang et al. [26] for some examples). 
Remark 4.5. Although Definition 4.1 is independent of the monotone condition, the verification of contracting rectangles often depends on the monotone condition. For example, if (F1) holds, then we can replace $f_{1}\left(a_{1}, b_{1}, b_{2}\right)>0$ by $f_{1}\left(a_{1}, r_{2}\left(z_{0}\right), s_{2}\left(z_{0}\right)\right)>0$ in Definition 4.1 , which can simplify the verification.

Remark 4.6. $\tau$ can be any positive constant.

\section{Applications}

In this part, we investigate the existence/nonexistence of traveling wave solutions of (1.4) with (1.6). Choose $m_{1}=m_{2}=2$, we can verify that (F1)(F4) hold. We further assume that (J1)-(J3), (K1)-(K4) hold in this section. Let

$$
u_{1}(x, t)=\phi_{1}(\xi), u_{2}(x, t)=\phi_{2}(\xi), \xi=x+c t
$$

be a traveling wave solution of (1.4). Then

$$
\left\{\begin{array}{l}
c \phi_{1}^{\prime}(\xi)=d_{1}\left[J_{1} * \phi_{1}\right](\xi)+F_{1}\left(\phi_{1}, \phi_{2}\right)(\xi), \xi \in \mathbb{R}, \\
c \phi_{2}^{\prime}(\xi)=d_{2}\left[J_{2} * \phi_{2}\right](\xi)+F_{2}\left(\phi_{1}, \phi_{2}\right)(\xi), \xi \in \mathbb{R},
\end{array}\right.
$$

in which $F_{1}\left(\phi_{1}, \phi_{2}\right)(\xi), F_{2}\left(\phi_{1}, \phi_{2}\right)(\xi)$ are defined by

$$
\begin{gathered}
F_{1}\left(\phi_{1}, \phi_{2}\right)(\xi)=r_{1} \phi_{1}(\xi)\left(1-\phi_{1}(\xi)-a_{1} \int_{\mathbb{R}} K_{1}(y) \phi_{1}\left(\xi-y-c \tau_{1}\right) d y\right. \\
\left.-b_{1} \int_{\mathbb{R}} K_{2}(y) \phi_{2}\left(\xi-y-c \tau_{2}\right) d y\right), \\
F_{2}\left(\phi_{1}, \phi_{2}\right)(\xi)=r_{2} \phi_{2}(\xi)\left(1-\phi_{2}(\xi)-a_{2} \int_{\mathbb{R}} K_{3}(y) \phi_{1}\left(\xi-y-c \tau_{3}\right) d y\right. \\
\left.-b_{2} \int_{\mathbb{R}} K_{4}(y) \phi_{2}\left(\xi-y-c \tau_{4}\right) d y\right) .
\end{gathered}
$$

For $c>0, \lambda>0$, define

$$
\begin{aligned}
& \Theta_{1}(\lambda, c)=d_{1}\left[\int_{\mathbb{R}} J_{1}(y) e^{\lambda y} d y-1\right]-c \lambda+r_{1}, \\
& \Theta_{2}(\lambda, c)=d_{2}\left[\int_{\mathbb{R}} J_{2}(y) e^{\lambda y} d y-1\right]-c \lambda+r_{2} .
\end{aligned}
$$

Lemma 5.1. There exists $c^{*}>0$ such that

(R1) if $c<c^{*}$, then $\Theta_{1}(\lambda, c)>0$ for any $\lambda>0$ or $\Theta_{2}(\lambda, c)>0$ for any $\lambda>0$

(R2) if $c>c^{*}$, there exist positive constants $\lambda_{1}(c), \lambda_{3}(c)$ such that

$$
\Theta_{1}(\lambda, c)\left\{\begin{array}{l}
=0, \lambda=\lambda_{1}(c), \lambda_{3}(c) \\
<0, \lambda \in\left(\lambda_{1}(c), \lambda_{3}(c)\right) \\
>0, \lambda \in\left(0, \lambda_{1}(c) \cup\left(\lambda_{3}(c),+\infty\right)\right.
\end{array}\right.
$$


(R3) if $c>c^{*}$, there exist positive constants $\lambda_{2}(c), \lambda_{4}(c)$ such that

$$
\Theta_{1}(\lambda, c)\left\{\begin{array}{l}
=0, \lambda=\lambda_{2}(c), \lambda_{4}(c), \\
<0, \lambda \in\left(\lambda_{2}(c), \lambda_{4}(c)\right), \\
>0, \lambda \in\left(0, \lambda_{2}(c) \cup\left(\lambda_{4}(c),+\infty\right) .\right.
\end{array}\right.
$$

Theorem 5.2. For each $c>c^{*}$, (5.1) has a positive solution $\left(\phi_{1}(\xi), \phi_{2}(\xi)\right)$ such that

$$
0<\phi_{i}(\xi)<1, \xi \in \mathbb{R}, \lim _{\xi \rightarrow-\infty} \phi_{i}(\xi) e^{-\lambda_{i}(c) \xi}=1, i=1,2 .
$$

Proof. We now prove the existence of traveling wave solutions by Theorem 3.2. Define

$$
\bar{\phi}_{i}(\xi)=\min \left\{e^{\lambda_{i}(c) \xi}, 1\right\}, \underline{\phi}_{i}(\xi)=\max \left\{e^{\lambda_{i}(c) \xi}-q e^{\lambda_{i}(c) \xi}, 0\right\}, i=1,2, \xi \in \mathbb{R},
$$

in which $q>1$ and $\eta>1$ such that

$$
\eta \in\left(1, \min \left\{\frac{\lambda_{1}(c)+\lambda_{2}(c)}{\lambda_{i}(c)}, \frac{\lambda_{i+2}(c)}{\lambda_{i}(c)}\right\}\right), i=1,2 .
$$

Let $q>1$ be

$$
\begin{aligned}
q= & +\frac{r_{1}+r_{1} a_{1} \int_{\mathbb{R}} K_{1}(y) e^{\lambda_{1}(y)} d y+r_{1} b_{1} \int_{\mathbb{R}} K_{2}(y) e^{\lambda_{2}(y)} d y}{-\Theta_{1}\left(\eta \lambda_{1}, c\right)} \\
& +\frac{r_{2}+r_{2} a_{2} \int_{\mathbb{R}} K_{3}(y) e^{\lambda_{1}(y)} d y+r_{2} b_{2} \int_{\mathbb{R}} K_{4}(y) e^{\lambda_{2}(y)} d y}{-\Theta_{2}\left(\eta \lambda_{2}, c\right)}
\end{aligned}
$$

then

$$
\left(\bar{\phi}_{1}(\xi), \bar{\phi}_{2}(\xi)\right),\left(\underline{\phi}_{1}(\xi), \underline{\phi}_{2}(\xi)\right)
$$

are a pair of upper and lower solutions of (5.1). The verification of the upper and lower solutions of (5.1) is evident, and we omit the details here.

By what we have done, we obtain the existence of $\left(\phi_{1}(\xi), \phi_{2}(\xi)\right)$, which is a solution to (5.1). The limit behavior of $\left(\phi_{1}(\xi), \phi_{2}(\xi)\right)$ is also clear. The proof is complete.

In particular, if

$$
a_{1}+b_{1}<1, a_{2}+b_{2}<1
$$

then (1.4) with (1.6) has a spatially homogeneous steady state defined by

$$
K=\left(k_{1}, k_{2}\right)=:\left(\frac{1+b_{2}-b_{1}}{\left(1+a_{1}\right)\left(1+b_{2}\right)-b_{1} a_{2}}, \frac{1+a_{1}-a_{2}}{\left(1+a_{1}\right)\left(1+b_{2}\right)-b_{1} a_{2}}\right) .
$$

Theorem 5.3. Assume that (5.2) holds. If $\left(\phi_{1}(\xi), \phi_{2}(\xi)\right)$ is formulated by Theorem 5.2, then $\left(\phi_{1}(\xi), \phi_{2}(\xi)\right)$ also satisfies

$$
\lim _{\xi \rightarrow \infty}\left(\phi_{1}(\xi), \phi_{2}(\xi)\right)=\left(k_{1}, k_{2}\right) .
$$


Proof. We now prove the result by Theorem 4.2. Note that a traveling wave solution $\phi_{1}(\xi)$ is smooth enough such that $u_{1}(x, t)=\phi_{1}(\xi)$ also satisfies

$$
\left\{\begin{array}{l}
\frac{\partial u_{1}(x, t)}{\partial t} \geq d_{1}\left[J_{1} * u_{1}\right](x, t)+r_{1} u_{1}(x, t)\left[1-a_{1}-b_{1}-u_{1}(x, t)\right], \\
u_{1}(x, 0)=\phi_{1}(x)>0, x \in \mathbb{R}
\end{array}\right.
$$

and

$$
\left\{\begin{array}{l}
\frac{\partial u_{1}(x, t)}{\partial t} \leq d_{1}\left[J_{1} * u_{1}\right](x, t)+r_{1} u_{1}(x, t)\left[1-u_{1}(x, t)\right] \\
u_{1}(x, 0)=\phi_{1}(x)>0, x \in \mathbb{R}
\end{array}\right.
$$

then Lemmas 2.2 and 2.4 imply that

$$
1 \geq \limsup _{t \rightarrow \infty} u_{1}(0, t) \geq \liminf _{t \rightarrow \infty} u_{1}(0, t) \geq 1-a_{1}-b_{1}>0,
$$

which further leads to

$$
1 \geq \limsup _{\xi \rightarrow \infty} \phi_{1}(\xi) \geq \liminf _{\xi \rightarrow \infty} \phi_{1}(\xi) \geq 1-a_{1}-b_{1}>0
$$

In a similar way, we have

$$
1 \geq \limsup _{\xi \rightarrow \infty} \phi_{2}(\xi) \geq \liminf _{\xi \rightarrow \infty} \phi_{2}(\xi) \geq 1-a_{2}-b_{2}>0 .
$$

Let

and define

$$
8 \epsilon=\min \left\{1-a_{1}-b_{1}, 1-a_{2}-b_{2}\right\}
$$

$$
\begin{array}{ll}
r_{1}(z)=z k_{1}, & r_{2}(z)=z k_{1}+(1-z)(1+\epsilon), \\
s_{1}(z)=z k_{2}, & s_{2}(z)=z k_{2}+(1-z)(1+\epsilon)
\end{array}
$$

for $z \in[0,1]$. By Smith [19, Section 5.7], we obtain a contracting rectangle of

$$
\left\{\begin{array}{l}
u_{1}^{\prime}(t)=r_{1} u_{1}(t)\left[1-u_{1}(t)-a_{1} u_{1}\left(t-\tau_{1}\right)-b_{1} u_{2}\left(t-\tau_{2}\right)\right], \\
u_{2}^{\prime}(t)=r_{2} u_{2}(t)\left[1-u_{1}(t)-a_{2} u_{1}\left(t-\tau_{3}\right)-b_{2} u_{2}\left(t-\tau_{4}\right)\right],
\end{array}\right.
$$

and there exists $z_{1} \in(0,1)$ such that

$$
\begin{aligned}
& 0<r_{1}\left(z_{1}\right)<1-a_{1}-b_{1}<1<r_{2}\left(z_{1}\right), \\
& 0<s_{1}\left(z_{1}\right)<1-a_{1}-b_{1}<1<s_{2}\left(z_{1}\right) .
\end{aligned}
$$

From Theorem 4.2, the proof is complete.

We now consider the nonexistence of traveling wave solutions by the idea in Lin and Ruan [12], and we first present the main conclusion as follows.

Theorem 5.4. If $c<c^{*}$, then (5.1) has no positive solution $\left(\phi_{1}(\xi), \phi_{2}(\xi)\right)$ satisfying

$$
(0,0) \ll\left(\phi_{1}(\xi), \phi_{2}(\xi)\right) \leq(1,1)
$$

and

$$
\lim _{\xi \rightarrow-\infty}\left(\phi_{1}(\xi), \phi_{2}(\xi)\right)=(0,0), \liminf _{\xi \rightarrow \infty}\left(\phi_{1}(\xi), \phi_{2}(\xi)\right) \gg(0,0) .
$$


Proof. Were the statement false, then there exists some $c_{1}<c^{*}$ such that (5.1) has a strictly positive solution $\left(\phi_{1}(\xi), \phi_{2}(\xi)\right)$ satisfying the restrictions in the theorem. Without loss of generality, we assume that

$$
\Theta_{1}\left(\lambda,\left(c_{1}+c^{*}\right) / 2\right)>0, \lambda \in(0, \infty) .
$$

Let

$$
\Theta_{1}^{\prime}(\lambda, c)=d_{1}\left[\int_{\mathbb{R}} J_{1}(y) e^{\lambda y} d y-1\right]-c \lambda+r_{1}[1-\varepsilon]
$$

with $\varepsilon>0$, then there exists

$$
\varepsilon_{0} \in\left(0, \min \left\{1-a_{1}-b_{1}, 1-a_{2}-b_{2}\right\}\right)
$$

such that for all $\varepsilon \in\left(0, \varepsilon_{0}\right]$, we have

$$
\Theta_{1}^{\prime}\left(\lambda,\left(c_{1}+c^{*}\right) / 2\right)>0, \lambda \in(0, \infty) .
$$

Choose $\xi_{0} \in \mathbb{R}, M>0$ such that

$$
\phi_{1}(\xi)<\frac{\varepsilon_{0}}{2}, \xi<\xi_{0}, \phi_{1}\left(\xi_{0}\right)=\frac{\varepsilon_{0}}{2},
$$

and

$$
\int_{-M+c \tau_{1}+c \tau_{2}}^{M-c \tau_{1}-c \tau_{2}} K_{1}(y)>1-\frac{\varepsilon_{0}}{8}, \int_{-M+c \tau_{1}+c \tau_{2}}^{M-c \tau_{1}-c \tau_{2}} K_{2}(y)>1-\frac{\varepsilon_{0}}{8} .
$$

Consider $\phi_{1}(\xi)$ for $\xi \in\left[\xi_{0}-M, \xi_{0}\right]$, then the continuity and the positivity imply that there exists $N>1$ such that

$$
\phi_{1}(\xi)>\frac{\varepsilon_{0}}{N}, \xi \in\left[\xi_{0}-M, \xi_{0}\right] .
$$

Moreover, let $N>1$ also satisfy

$$
\phi_{1}(\xi)>\frac{\varepsilon_{0}}{N}, \phi_{2}(\xi)>\frac{\varepsilon_{0}}{N}, \xi \in\left[\xi_{0}-M, \infty\right) .
$$

By the limit behavior of $\xi \rightarrow \infty$, we see that $N$ is admissible.

If $\xi \leq \xi_{0}-M$, then

$1-\phi_{1}(\xi)-a_{1} \int_{\mathbb{R}} K_{1}(y) \phi_{1}\left(\xi-y-c \tau_{1}\right) d y-b_{1} \int_{\mathbb{R}} K_{2}(y) \phi_{2}\left(\xi-y-c \tau_{2}\right) d y \geq 1-\varepsilon_{0}$.

For $\xi>\xi_{0}-M$, we have

$$
\begin{aligned}
& 1-\phi_{1}(\xi)-a_{1} \int_{\mathbb{R}} K_{1}(y) \phi_{1}\left(\xi-y-c \tau_{1}\right) d y-b_{1} \int_{\mathbb{R}} K_{2}(y) \phi_{2}\left(\xi-y-c \tau_{2}\right) d y \\
\geq & 1-\frac{\varepsilon_{0}}{2}-\left(1+\left(a_{1}+b_{1}\right) \frac{N}{\varepsilon_{0}}\right) \phi_{1}(\xi) .
\end{aligned}
$$

Therefore, $\phi_{1}(\xi), \phi_{2}(\xi)$ satisfies

$$
\begin{aligned}
& 1-\phi_{1}(\xi)-a_{1} \int_{\mathbb{R}} K_{1}(y) \phi_{1}\left(\xi-y-c \tau_{1}\right) d y-b_{1} \int_{\mathbb{R}} K_{2}(y) \phi_{2}\left(\xi-y-c \tau_{2}\right) d y \\
\geq & 1-\varepsilon_{0}-\left(1+\left(a_{1}+b_{1}\right) \frac{N}{\varepsilon_{0}}\right) \phi_{1}(\xi), \xi \in \mathbb{R},
\end{aligned}
$$


and so $\phi_{1}(\xi)=u_{1}(x, t)$ leads to

$\left\{\begin{array}{l}\frac{\partial u_{1}(x, t)}{\partial t} \geq d_{1}\left[J_{1} * u_{1}\right](x, t)+r_{1} u_{1}(x, t)\left[1-\varepsilon_{0}-\left(1+\left(a_{1}+b_{1}\right) \frac{N}{\varepsilon_{0}}\right) u_{1}(x, t)\right], \\ u_{1}(x, 0)=\phi_{1}(x)>0 .\end{array}\right.$

From Lemmas 2.2 and 2.4, we obtain

$$
\liminf _{t \rightarrow \infty} \inf _{2|x|=\left(c^{\prime}+c^{*}\right) t} u_{1}(x, t) \geq \frac{1-\varepsilon_{0}}{1+\left(a_{1}+b_{1}\right) \frac{N}{\varepsilon_{0}}}>0 .
$$

Let $-2 x=\left(c^{\prime}+c^{*}\right) t$. Then

$$
\xi=x+c_{1} t \rightarrow-\infty, t \rightarrow \infty
$$

and

$$
\lim _{\xi \rightarrow-\infty} \phi_{1}(\xi)=\lim _{t \rightarrow \infty} u_{1}\left(-\left(c^{\prime}+c^{*}\right) t / 2, t\right)=0,
$$

which implies a contradiction. The proof is complete.

\section{References}

[1] P. W. Bates, On some nonlocal evolution equations arising in materials science, In: Nonlinear dynamics and evolution equations (Ed. by H. Brunner, X. Zhao and X. Zou), pp. 13-52, Fields Inst. Commun. 48, AMS, Providence, 2006.

[2] P. W. Bates, P. C. Fife, X. Ren, and X. Wang, Traveling waves in a convolution model for phase transitions, Arch. Rational Mech. Anal. 138 (1997), no. 2, 105-136.

[3] J. Carr and A. Chmaj, Uniqueness of travelling waves for nonlocal monostable equations, Proc. Amer. Math. Soc. 132 (2004), no. 8, 2433-2439.

[4] X. Chen, Existence, uniqueness, and asymptotic stability of traveling waves in nonlocal evolution equations, Adv. Differential Equations 2 (1997), no. 1, 125-160.

[5] J. Coville and L. Dupaigne, Propagation speed of travelling fronts in non local reactiondiffusion equations, Nonlinear Anal. 60 (2005), no. 5, 797-819.

[6] - On a non-local equation arising in population dynamics, Proc. Roy. Soc. Edinburgh Sect. A 137 (2007), no. 4, 727-755.

[7] S. A. Gourley and J. Wu, Delayed non-local diffusive systems in biological invasion and disease spread, In: Nonlinear dynamics and evolution equations (Ed. by H. Brunner, X. Zhao and X. Zou), pp. 137-200, Fields Inst. Commun. 48, AMS, Providence, 2006.

[8] L. Hopf, Introduction to Differential Equations of Physics, Dover, New York, 1948.

[9] Y. Jin and X. Q. Zhao, Spatial dynamics of a periodic population model with dispersal, Nonlinearity 22 (2009), no. 5, 1167-1189.

[10] W. T. Li, Y. Sun, and Z. C. Wang, Entire solutions in the Fisher-KPP equation with nonlocal dispersal, Nonlinear Anal. Real World Appl. 11 (2010), no. 4, 2302-2313.

[11] X. Liang and X. Q. Zhao, Asymptotic speeds of spread and traveling waves for monotone semiflows with applications, Comm. Pure Appl. Math. 60 (2007), no. 1, 1-40.

[12] G. Lin and S. Ruan, Traveling wave solutions for delayed reaction-diffusion systems and applications to Lotka-Volterra competition-diffusion models with distributed delays, J. Dynam. Diff. Eqns., in press, DOI: 10.1007/s10884-014-9355-4.

[13] J. D. Murray, Mathematical Biology, Springer, Berlin-Heidelberg-New York, 1993.

[14] S. Pan, Traveling wave fronts of delayed non-local diffusion systems without quasimonotonicity, J. Math. Anal. Appl. 346 (2008), no. 2, 415-424.

[15] S. Pan, W. T. Li, and G. Lin, Travelling wave fronts in nonlocal delayed reactiondiffusion systems and applications, Z. Angew. Math. Phys. 60 (2009), no. 3, 377-392. 
[16] _ Existence and stability of traveling wavefronts in a nonlocal diffusion equation with delay, Nonlinear Anal. 72 (2010), no. 6, 3150-3158.

[17] W. Shen and A. Zhang, Spreading speeds for monostable equations with nonlocal dispersal in space periodic habitats, J. Differential Equations 249 (2010), no. 4, 747-795.

[18] N. Shigesada and K. Kawasaki, Biological Invasions: Theory and Practice, Oxford University Press, Oxford, 1997.

[19] H. L. Smith, Monotone Dynamical Systems: An Introduction to the Theory of Competitive and Cooperative Systems, AMS, Providence, RI, 1995.

[20] Y. Sun, W. T. Li, and Z. C. Wang, Traveling waves for a nonlocal anisotropic dispersal equation with monostable nonlinearity, Nonlinear Anal. 74 (2011), no. 3, 814-826.

[21] S. Wu and S. Liu, Traveling waves for delayed non-local diffusion equations with crossing-monostability, Appl. Math. Comput. 217 (2010), no. 4, 1435-1444.

[22] J. Xia, Z. Yu, and R. Yuan, Traveling waves of a competitive Lotka-Volterra model with nonlocal diffusion and time delays, Acta Math. Appl. Sin. 34 (2011), no. 6, 1082-1093.

[23] $\mathrm{Z}$. Xu and P. Weng, Traveling waves in a convolution model with infinite distributed delay and non-monotonicity, Nonlinear Anal. Real World Appl. 12 (2011), no. 1, 633647.

[24] Z. Yu and R. Yuan, Travelling wave solutions in nonlocal reaction-diffusion systems with delays and applications, ANZIAM J. 51 (2009), no. 1, 49-66.

[25] — Travelling wave solutions in non-local convolution diffusive competitivecooperative systems, IMA J. Appl. Math. 76 (2011), no. 4, 493-513.

[26] G. Zhang, W. T. Li, and G. Lin, Traveling waves in delayed predator-prey systems with nonlocal diffusion and stage structure, Math. Comput. Modelling 49 (2009), no. 5-6, 1021-1029.

[27] G. Zhang, W. T. Li, and Z. C. Wang, Spreading speeds and traveling waves for nonlocal dispersal equations with degenerate monostable nonlinearity, J. Differential Equations 252 (2012), no. 9, 5096-5124.

Department of Applied Mathematics LANZHOU UNIVERSiTY OF TECHNOLOGY LANZhou, Gansu 730050, P. R. China

E-mail address: shxpan@yeah.net 